\title{
Pre-metastatic conditioning of organ microenvironments by tumors: beyond preparing the soil
}

\author{
Jonathan P. Sleeman ${ }^{1,2}$
}

Published online: 31 October 2015

(C) Springer-Verlag Berlin Heidelberg 2015

Paget's "seed and soil" hypothesis to explain patterns of cancer metastasis [1] must rank as the longest-enduring and most influential concept in oncology. Nevertheless, our understanding of what defines a metastatic "seed" as well as the underlying cell and molecular basis of what constitutes a receptive microenvironmental "soil" is clearly only partial and continues to evolve to this day. For example, ideas about hierarchical tumor cell subpopulations founded on cancer stem cells clearly have important implications about whether all or only some tumor cells can act as seeds during metastasis formation [2].

With regard to the soil in which metastases develop, recent years have witnessed rapid progress, and it is now clear that the nature of the soil is dynamic and can be influenced by a number of factors, including the primary tumor itself. In particular, seminal work from David Lyden's laboratory 10 years ago [3] has stimulated intensive work into defining what constitutes a metastatic niche - an organ microenvironment that supports the survival and outgrowth of disseminated tumor cells - and how factors released by tumors can condition organ microenvironments to create pre-metastatic niches in advance of tumor cell dissemination.

The notion that tumor-derived factors can stimulate metastasis formation harks back to early ideas about how cancer dissemination occurs. Prior to the acceptance of cell theory as the basis of life and its application to pathology, various theories postulated that cancer was spread by poisonous or infectious "juices" produced by tumors that caused transformation at secondary sites, leading to metastasis formation [4]. While metastasis is clearly

Jonathan P. Sleeman

Jonathan.Sleeman@medma.uni-heidelberg.de

1 University of Heidelberg, Medical Faculty Mannheim, (CBTM), TRIDOMUS-Gebäude Haus C, Ludolf-Krehl-Str. 13-17, 68167 Mannheim, Germany

2 Karlsruhe Institute for Technology (KIT), Campus Nord, Institut für Toxikologie und Genetik, Postfach 3640, 76021 Karlsruhe, Germany not caused by secondary transformation but rather by dissemination of tumor cells from the primary cancer, nevertheless, it has emerged that factors that are shed and secreted by primary tumors can be decisive in metastasis formation through conditioning organ microenvironments in which metastases ultimately develop. Understanding these tumor-derived juices and the critical components within them is thus clearly an additional important aspect to be considered alongside the seed and soil and is obviously highly relevant therapeutically.

Pre-metastatic conditioning likely contributes to a number of observations regarding metastasis formation. The concentration of factors produced by tumors that can induce a pre-metastatic niche in future sites of metastasis must presumably exceed a given threshold in order to condition the target organ microenvironment. This presumably contributes to the fact that the size of a primary tumor often correlates with the risk of metastasis formation [5]. Furthermore, as tumor-draining lymph nodes are exposed to high concentrations of metastatic niche-conditioning factors that are present in the lymphatic fluid they receive from the primary tumor, this must contribute at least in part to the prevalence of lymph node metastases for many types of cancer [6]. Moreover, some tumors such as melanoma metastasize while the primary tumor is still very small, while other tumors such as basal cell carcinoma can grow to large sizes without metastasizing. In the future, it will be interesting to explore whether the intrinsic metastatic potential of particular types of cancer is due to different expression levels of key premetastatic niche-inducing factors in the primary tumor. In addition, it is interesting to speculate whether skeletal muscle is rarely a site for metastasis formation because it does not respond to premetastatic conditioning or whether the reason why particular types of cancer metastasize to unusual organs (for example thyroid metastasis formation by clear cell kidney carcinomas) is due in part to the particular spectrum of factors these types of cancer produce that can pre-metastatically condition the organ concerned and make it receptive for disseminating tumor cells.

Pre-metastatic conditioning of putative sites of metastasis formation may also contribute to the observation that metastases 
often form in the next organ that receives blood flow from a primary tumor. Traditionally, the anatomical/mechanical theory has been used to explain these patterns of metastatic spread, which postulates that mechanical entrapment of circulating tumor cells explains why metastases often form in these organs [5]. However, it is notable that these organs will also receive higher concentration of factors from primary tumors that can pre-metastatically condition the organ microenvironment, which may also contribute to these patterns of metastatic spread. Similar considerations may also explain why the lung is a prevalent site of metastasis for many types of cancer, because lymphatic drainage patterns determine that the lung in particular is exposed to increasingly high levels of metastatic niche-conditioning factors as primary tumors grow and progress. Specifically, after being transported through the lymphatic system, lymphatic fluid draining from primary tumors enters the blood stream mainly through the thoracic duct and into the left subclavian vein. This lymph-rich blood then passes through the right ventricle of the heart before entering the pulmonary arteries and ultimately the lung capillaries. Thus, of all organs except the lymph nodes, the lung likely receives the highest concentration of factors from primary tumors that can induce the formation of pre-metastatic niches.

Pre-metastatic conditioning is unlikely to be the only way in which endogenous organ microenvironments are modified to create effective metastatic niches. Metastatic recurrence often follows a long latency period where metastases become clinically apparent many years after removal of the primary tumor and adjuvant therapy, because even though the primary disease may have been diagnosed at an early stage, dormant tumor cells have already disseminated to future metastatic sites [7, 8]. Little is known about what reactivates dormant tumor cells, although remodelling of the ECM and the presence of certain types of immune cell have been implicated in the transition from dormancy to metastatic growth [9], suggesting that post-dissemination changes in organ microenvironments triggered by as yet largely unidentified factors may create metastatic niches that stimulate outgrowth of previously dormant tumor cells.

The concept of the metastatic niche draws on findings made at several organ sites. Nevertheless, different types of cancer show a predilection to metastasize to different organs. This presumably reflects not only the differing microenvironments intrinsically offered by each type of organ and intrinsic tumor cell properties that are characteristic for a given type of cancer that allows them to interact with a particular organ microenvironment, but also that different factors that metastatically condition particular organs are produced by the type of cancer concerned or by dormancybreaking events.

Focusing on the lymph nodes, lung, liver, bone marrow, and brain as major organs in which metastases often develop, the aim of this review series is to explore our current understanding of organ-specific metastatic niches, focusing on how the underlying endogenous organ microenvironment and metastatic conditioning combine to produce a competent organ- specific metastatic niche. For the brain and bone, it is clear that much work remains to be done to determine whether premetastatic conditioning is relevant at these sites, and if so, what form it takes and how it is regulated $[10,11]$. Several features appear to be common between metastatic niches in different organs, in particular the creation of an immunosuppressive microenvironment appears essential for effective metastatic niche function in organs such as the lung, liver, and lymph node $[6,12,13]$. Nevertheless, clear differences between metastatic niches in different organs are also apparent. For example, due to its role in immunity, pre-metastatic changes in the lymph node counter-intuitively often initially restrict metastatic growth [6]. Cells unique to the liver such as hepatic sinusoidal endothelial cells, hepatocytes, and hepatic stellate cells make a major contribution to the microenvironmental support of metastatic outgrowth in the liver [12]. The osteoblastic niche can provide a ready-made metastatic niche for tumor cells that disseminate to the bone [11]. Further research aimed at understanding features of metastatic niches unique to particular organs will not only explain Paget's seed and soil theory at the cellular and molecular levels but will also identify novel targets that can be targeted therapeutically.

\section{References}

1. Paget S (1889) The distribution of secondary growths in cancer of the breast. Lancet I:571-573

2. Sleeman JP, Cremers N (2007) New concepts in breast cancer metastasis: tumor initiating cells and the microenvironment. Clin Exp Metastasis 24:707-715

3. Kaplan RN, Riba RD, Zacharoulis S, Bramley AH, Vincent L, Costa C, MacDonald DD, Jin DK, Shido K, Kerns SA et al (2005) VEGFR1-positive haematopoietic bone marrow progenitors initiate the pre-metastatic niche. Nature 438:820-827

4. Weiss L (2000) Metastasis of cancer: a conceptual history from antiquity to the 1990s. Cancer Metastasis Rev 19(I-XI):193-383

5. Sleeman JP (2000) The lymph node as a bridgehead in the metastatic dissemination of tumors. Recent Results Cancer Res 157:55-81

6. Sleeman JP (2015) The lymph node pre-metastatic niche. J Mol Med. doi:10.1007/s00109-015-1351-6

7. Sleeman JP, Nazarenko I, Thiele W (2011) Do all roads lead to Rome? Routes to metastasis development. Int J Cancer J Int du Cancer 128:2511-2526

8. Uhr JW, Pantel K (2011) Controversies in clinical cancer dormancy. Proc Natl Acad Sci U S A 108:12396-12400

9. Sleeman JP, Christofori G, Fodde R, Collard JG, Berx G, Decraene C, Ruegg C (2012) Concepts of metastasis in flux: the stromal progression model. Semin Cancer Biol 22:174-186

10. Winkler F (2015) The brain metastatic niche. J Mol Med. doi:10. 1007/s00109-015-1357-0

11. Ren G, Esposito M, Kang Y (2015) Bone metastasis and metastatic niche. J Mol Med. doi:10.1007/s00109-015-1329-4

12. Krüger A (2015) Premetastatic niche formation in the liver: emerging mechanisms and mouse models. J Mol Med. doi:10.1007/ s00109-015-1342-7

13. Maru Y (2015) The lung metastatic niche. J Mol Med. doi: 10.1007/s00109-015-1355-2 